\title{
Testosterone supplementation and body composition: results from a meta- analysis of randomized controlled trials.
}

Giovanni Corona ${ }^{1}$, Vito A Giagulli², Elisa Maseroli ${ }^{3}$, Linda Vignozzi ${ }^{3}$, Antonio Aversa ${ }^{4}$, Michael Zitzmann ${ }^{5}$, Farid Saad $^{6}$, Edoardo Mannucci ${ }^{7}$ and Mario Maggi ${ }^{3}$

\begin{abstract}
${ }^{1}$ Endocrinology Unit, Medical Department, Azienda Usl Bologna Maggiore-Bellaria Hospital, Bologna, Italy, ${ }^{2}$ Unit of Metabolic Diseases and Endocrinology, Conversano, Italy; ${ }^{3}$ Andrology and Sexual Medicine Unit, Department of Experimental and Clinical Biomedical Sciences, University of Florence, Florence, Italy; ${ }^{4}$ Department of Experimental Medicine, Sapienza University of Rome, Rome, Italy; ${ }^{5}$ Centre for Reproductive Medicine and AndrologyMuenster, Germany; ${ }^{6}$ Bayer Pharma, Global Medical Affairs Andrology, Berlin, Germany and Gulf Medical University School of Medicine, Ajman, United Arab Emirates; ${ }^{7}$ Diabetes Agency, Careggi Hospital, Florence, Italy
\end{abstract}

Objective. The role of testosterone $(T)$ in regulating body composition is conflicting. The aim of present study is too meta-analyze the effects of $\mathrm{T}$ supplementation (TS) on body composition and metabolic outcomes
Methods. All randomized controlled trials (RCTs) comparing the effect of TS on different endpoints were considered.

\section{Results}

Overall, 59 trials were included in the study enrolling 3029 and 2049 patients in TS and control groups, respectively. Mean trial duration was 8.7 months. The mean age, baseline $T$ and body mass index of enrolled patients were 62.0 years, $11.6 \mathrm{nmoles} / \mathrm{L}$ and $28.6 \mathrm{~kg} / \mathrm{m}^{2}$ respectively.

Trial flow diagram

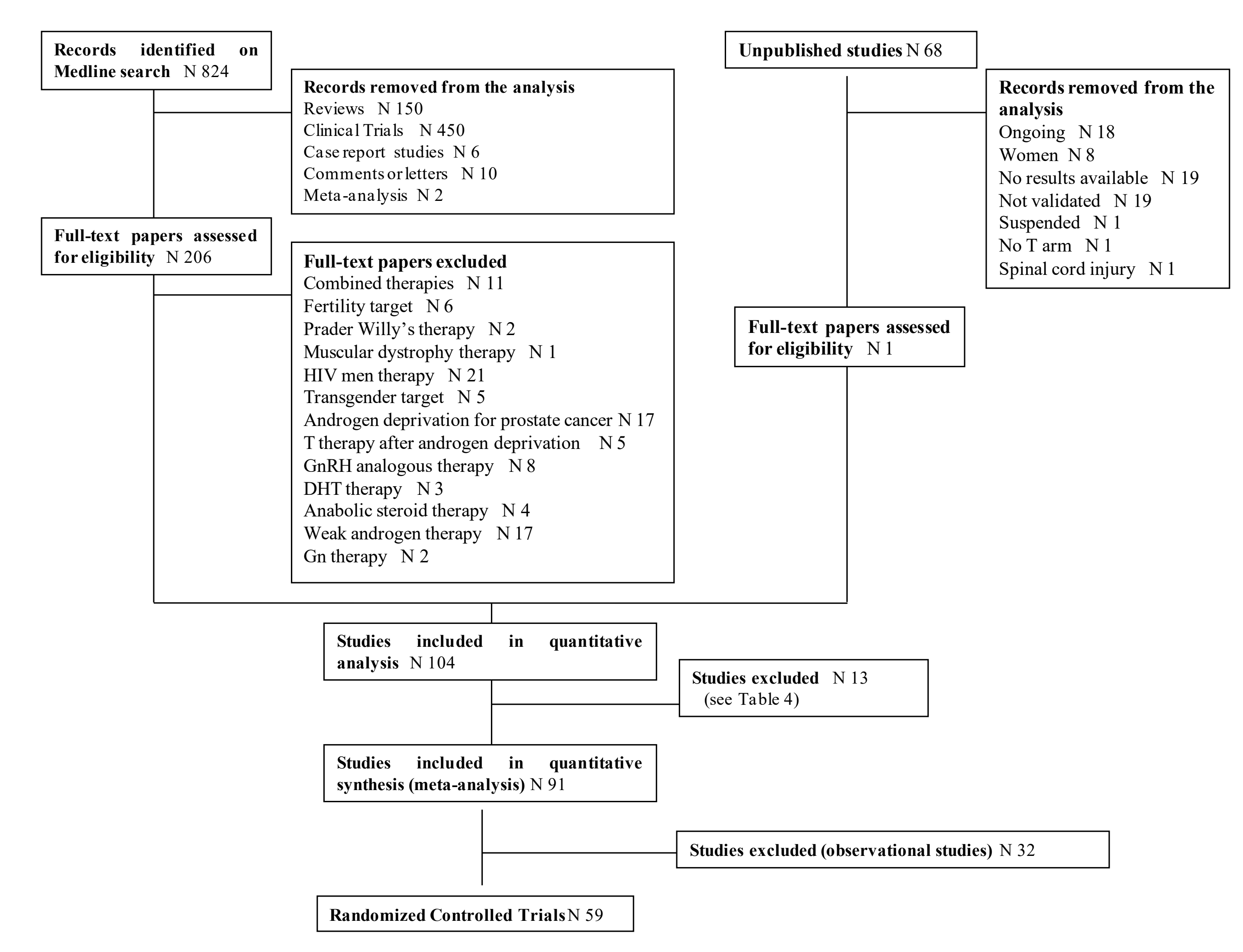

Weighted mean differences (with 95\%CI) of different parameters at end point

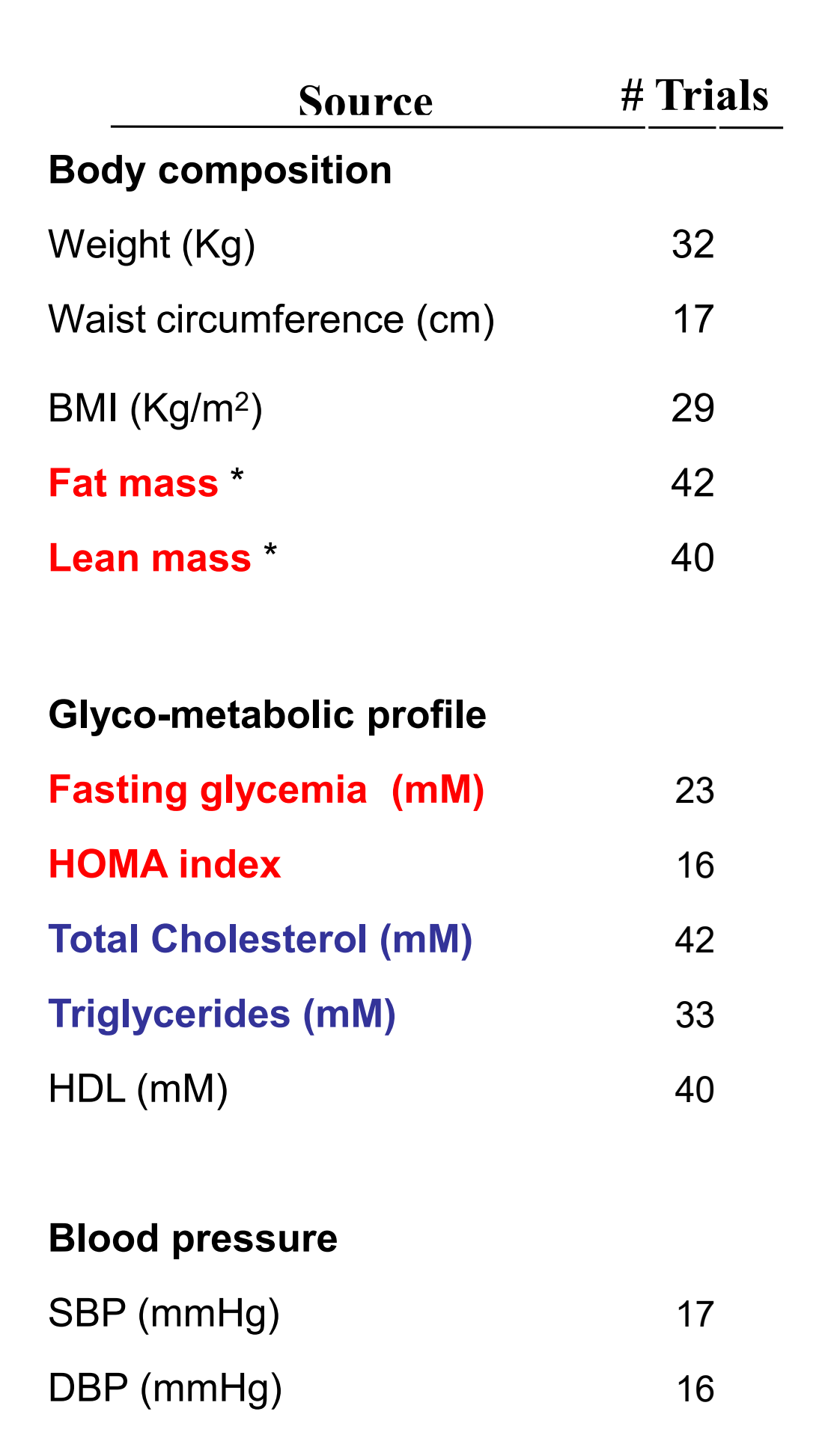

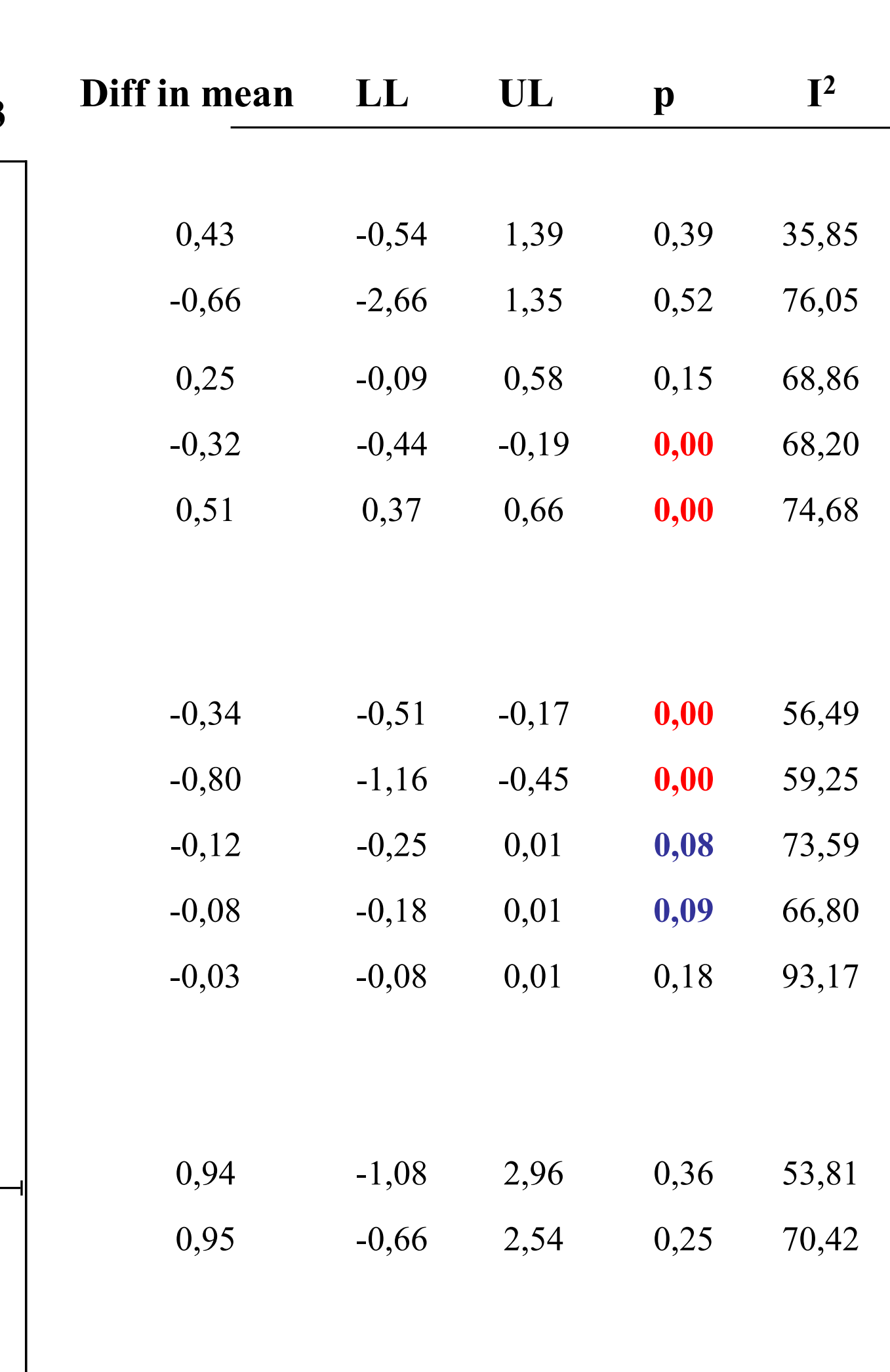

End-point testosterone level modification adjusted relationship between TSinduced glycol-metabolic improvement and body composition changes

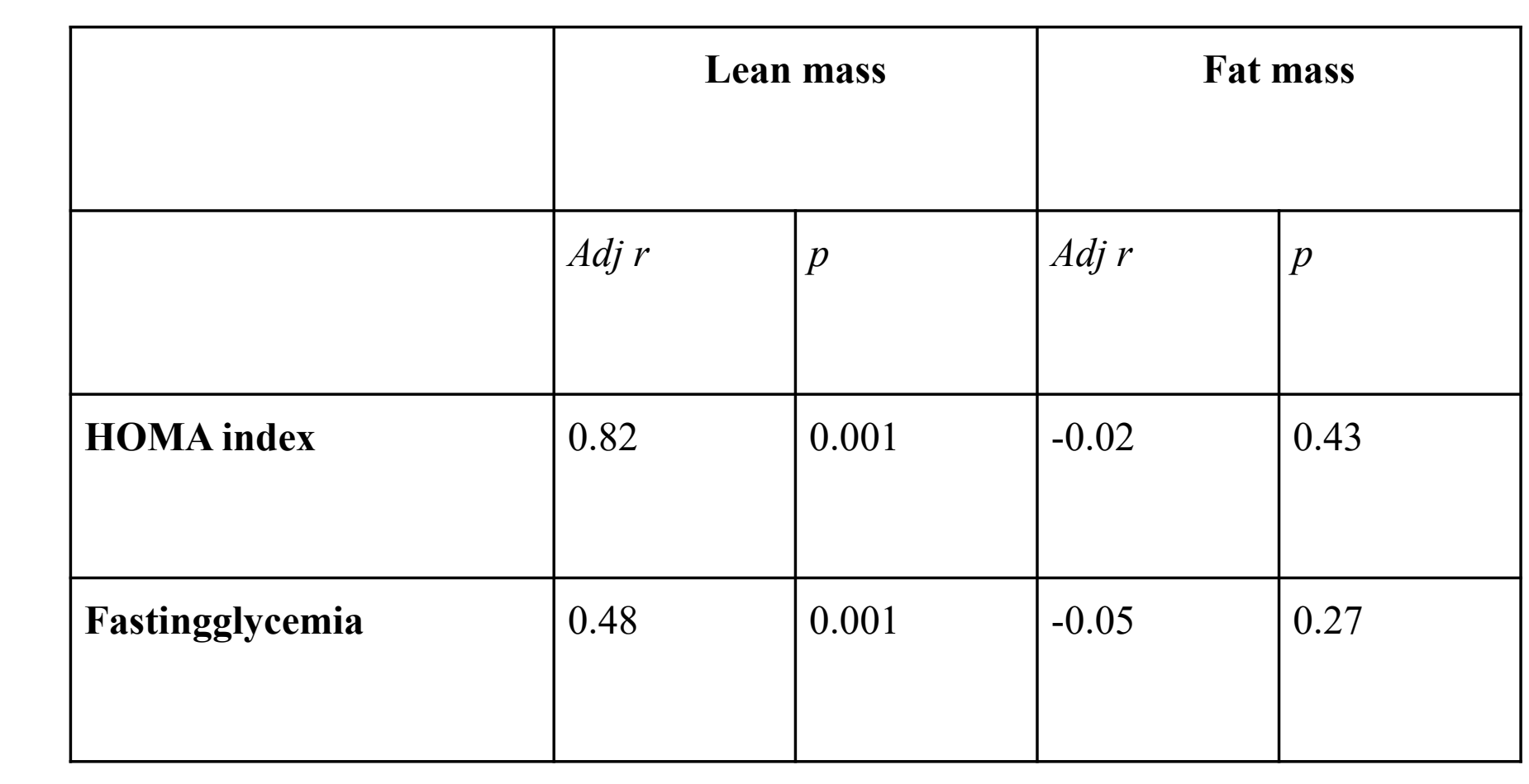

Conclusions. Our data suggest that TS is able to improve body composition and glycometabolic profile particularly in younger subjects and in those with metabolic disturbances. Specifically designed studies are urgently needed to confirm this point. 